\title{
INQUIETUDES Y COMPLICIDADES EN EL USO DEL SOFTWARE DE GESTIÓN EN CENTROS EDUCATIVOS.
}

Resumen: El nivel de introducción del denominado software de gestión en los centros de enseñanza secundaria ya es muy elevado, y en consecuencia susceptible de ser analizado. En estas páginas, en base a los primeros resultados de una investigación que estamos realizando en la Comunidad Valenciana, reflexionamos sobre el uso en algunos de estos centros de aplicaciones y artefactos de gestión, destacando las tensiones que se perciben entre sus posibilidades como recurso de ayuda al profesorado, y los condicionamientos que como instrumento de control impone su utilización.

Palabras clave: Software de gestión, organización escolar, centros de secundaria, TIC, instrumentos de control.

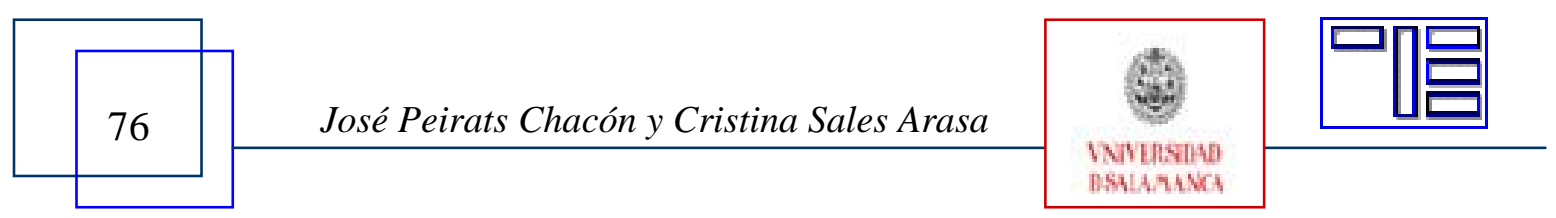




\title{
WORRIES AND COMPLICITIES IN THE USE OF THE SOFTWARE OF MANAGEMENT IN EDUCATIONAL CENTERS.
}

\begin{abstract}
The level of introduction of the so called software of management in the educational centers of secondary education is already very high, and consequently capable of being analyzed. On these pages, based on the first results of an investigation that we realize in the Valencian Community, we reflect on the use in some of these centers of applications and gadgetry of management, emphasizing the tensions that are perceived among its possibilities as a resource of help to the professorship, and the conditionings its use imposes as an instrument of control.
\end{abstract}

Keywords: Software of management, school organization, centers of secondary, Information and Communication Technologies, instruments of control.

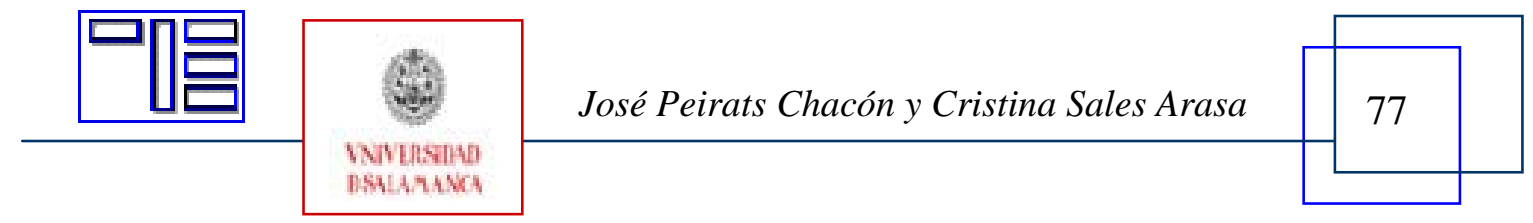


Revista Electrónica Teoría de la Educación.

Educación y Cultura en la Sociedad de la Información.

http://www.usal.es/teoriaeducacion

Vol. 10. No1. Marzo 2009

\section{DES PRÉOCCUPATIONS ET DES COMPLICITÉS DANS L'USAGE DU SOFTWARE DE GESTION À DES CENTRES ÉDUCATIFS.}

Sommaire : Le niveau d'introduction du soi-disant software de gestion aux centres d'enseignement secondaire est déjà très élevé, et en conséquence susceptible d'être analysé. À ces pages, à coups des premiers résultats d'une recherche que nous réalisons dans la Communauté Valencienne, nous réfléchissons à l'usage dans certains de ces centres des applications et d'engins de gestion, en détachant les tensions qui sont perçues entre leurs possibilités comme recours d'aide au professorat, et les conditions que, comme instrument de contrôle, impose leur utilisation.

Mots clefs: Software de gestion, organisation scolaire, centres de secondaire, technologies de l'Information et de la Communication, instruments de contrôle.

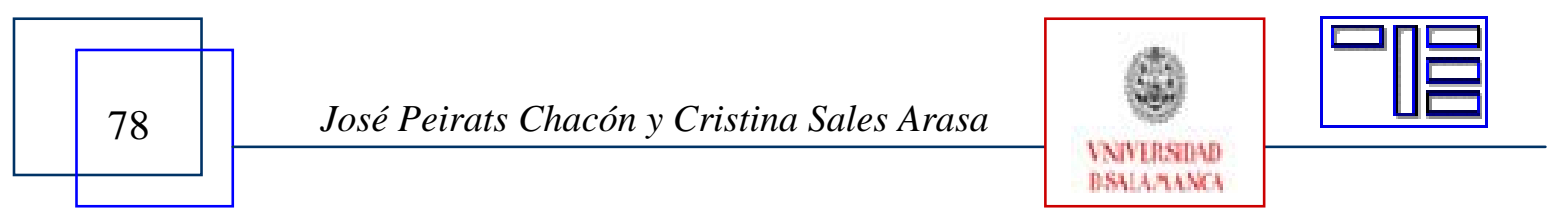




\begin{tabular}{|c|c|c|}
\hline \multirow[t]{2}{*}{ 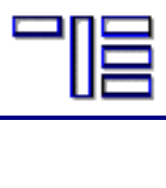 } & \multicolumn{2}{|c|}{$\begin{array}{l}\text { Revista Electrónica Teoría de la Educación. } \\
\text { Educación y Cultura en la Sociedad de la Información. }\end{array}$} \\
\hline & http://www.usal.es/teoriaeducacion & Vol. 10. No 1. Marzo 2009 \\
\hline
\end{tabular}

\title{
INQUIETUDES Y COMPLICIDADES EN EL USO DEL SOFTWARE DE GESTIÓN EN CENTROS EDUCATIVOS.
}

\author{
José Peirats Chacón (Pepe.Peirats@uv.es) \\ Cristina Sales Arasa (Cristina.Sales@uv.es) \\ Universitat de València
}

\section{1.- INTRODUCCIÓN.}

La gestión de centros docentes a través de medios tecnológicos, aunque ya no es un aspecto novedoso, sí que está cobrando un interés cada vez mayor en el debate general sobre la organización de los centros. En los últimos años han ido convergiendo progresivamente más y más tecnologías en las instituciones educativas, sustituyendo los anteriores procedimientos basados en los medios impresos por artefactos tecnológicos que procuran la gestión y la comunicación instantánea de los datos. Y no sólo ha ocurrido esto en los centros educativos sino que es una tendencia observable en la mayoría de las organizaciones actuales. Se habla de organizaciones que aprenden y parece que este término va unido a la gestión del conocimiento a través de sistemas y tecnologías de la información. Bill Gates afirmaba que le gestión del conocimiento no es nada más que "gerenciar" el flujo de la información, hacer llegar la información correcta a la gente que la necesita, de tal manera que puede actuar con rapidez (Dessler, 2001, 234). ¿Apuntará también a este fin la gestión de centros escolares a través de TIC? Autores como Llorens i Cerdà $(2003,1)$ responden que en el marco de los entornos educativos no tiene por qué suceder algo distinto a lo que ocurre en otras organizaciones.

La Administración educativa lo justifica argumentando la necesidad de disminuir el elevado número de actividades administrativas de los centros y de facilitar las labores de los equipos directivos. La empresa privada también se apunta por motivos obviamente comerciales a esta tendencia, ofertando programas de gestión diversos. También hay voces que señalan la adaptación que la escuela tiene que realizar a las innovaciones y cambios que tienen lugar en la sociedad; Ramírez por ejemplo afirma $(2004,134)$ que "las transformaciones experimentadas en lo social, en lo político, en lo económico, en lo comercial, en lo científico, en lo cultural, y en todas las demás dimensiones de la vida

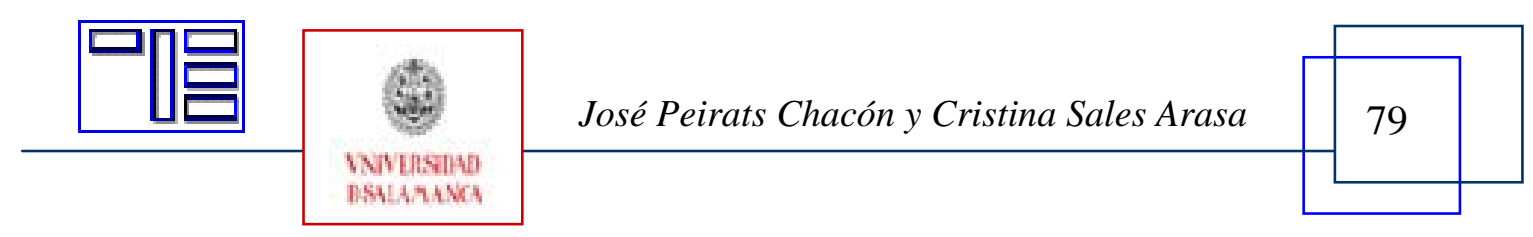


Revista Electrónica Teoría de la Educación.

Educación y Cultura en la Sociedad de la Información.

http://www.usal.es/teoriaeducacion

Vol. 10. No1. Marzo 2009

moderna deben ser asumidas proactivamente por los educadores y por los directivos de las instituciones educativas, para anticiparse al futuro".

En cualquier caso, más allá de las razones que se esgrimen, la realidad es que la aplicación de las TIC a la gestión de centros escolares está causando auténticos quebraderos de cabeza en las secretarías de los centros. Y creemos que esto ocurre porque no sólo se trata de adoptar nuevas herramientas o nuevos programas para la gestión; la cuestión es de mayor importancia puesto que la relación TIC-organización escolar va más allá de cuestiones instrumentales, generando nuevas interacciones entre los miembros de la comunidad educativa y por qué no decirlo, nuevos e inevitables conflictos. Aunque considerados desde una visión positiva, al poder "ser un factor fundamental para el cambio y el desarrollo organizativo de los centros de carácter democrático, participativo y colaborativo" (Jares, 1997, 15).

Bien es cierto que el incremento de las actividades administrativas en los centros, a pesar de las reiteradas promesas de agilización o eliminación por la política partidaria de turno en las instancias de poder, es una realidad que nadie cuestiona. Los equipos directivos de los centros se quejan de la falta de recursos administrativos para atender las continuas peticiones de los padres, profesores, alumnos e incluso de la propia Administración en relación con los procesos que acontecen a lo largo del curso escolar: tramitación de becas de libros, comedor, transporte escolar, traslados de alumnos, expedición de todo tipo de certificados, elaboración de la Programación Anual, Memoria, etc. y prueba de ello la encontramos en la prensa diaria. En este conjunto de tareas no hay que olvidar que los centros de secundaria cuentan, además del secretario del equipo directivo, con personal administrativo en plantilla que asume o ayuda en las labores anteriormente citadas; sin embargo, en los centros de primaria esta figura sólo la podemos encontrar en contados casos, en grandes centros de tres o más líneas educativas o con una problemática especial, y que ha sido repetidamente reclamada por los sindicatos y por las asociaciones de directores.

Es en esta perspectiva donde han surgido con fuerza en los últimos tiempos numerosas tecnologías de carácter informático, tanto en las instancias de la Administración educativa como de la empresa privada, que pretenden ayudar, complementar o solucionar los problemas de gestión en los centros docentes. Ahora bien ¿cómo se está entendiendo la gestión? Pensamos, al igual que Ezpeleta (1997) que muchas veces "se interpreta como el transplante de saberes propios del orden de la economía sólo para potenciar la perspectiva administrativa, o bien para pensar al sistema y sus escuelas como réplicas de la empresa”. De hecho, estas tecnologías son producto, muchas de ellas, de las políticas diseñadas e impulsadas en los últimos años sobre la e-Administración ${ }^{[1]}$, facilitando a los clientes la interacción con las organizaciones, flexibilizando y mejorando las condiciones de trabajo de los empleados, pero sin estar pensadas ex profeso para el ámbito educativo.

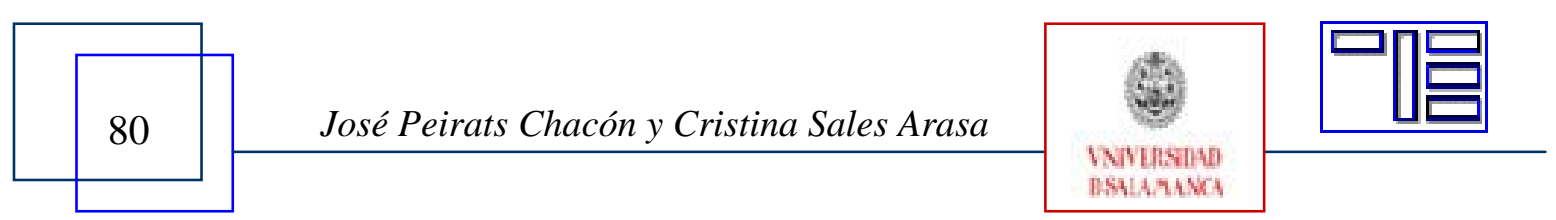


Por tanto, la gestión en las escuelas no sólo es administrativa sino también política y esto es algo que vamos a mostrar en las siguientes líneas. Describiremos algunos de los sistemas de gestión que se están introduciendo en los centros educativos del ámbito territorial valenciano, y pondremos en evidencia las dificultades que se encuentran y las contradicciones y conflictos que se generan al intentar que la escuela sea una organización que a través de las TIC, por utilizar los términos que están en boga, gestione el conocimiento.

Estas reflexiones se enmarcan dentro de una de las líneas de investigación del Grupo de Investigación $\mathrm{CRIE}^{[2]}$, la que se ocupa del estudio de las implicaciones de la gestión digital en los centros docentes. En ese ámbito estamos trabajando en un proyecto de investigación en centros de enseñanza secundaria de la Comunidad Valenciana; este artículo presenta algunas de las conclusiones provisionales del estudio.

\section{2.- LOS SISTEMAS DE GESTIÓN EN LOS CENTROS EDUCATIVOS.}

En los momentos actuales, en cualquiera de las dependencias del centro -biblioteca, aulas especiales, laboratorios, etc.- podemos encontrar tecnologías informáticas. Si McFarlane $(2001,104)$ mostraba no hace mucho su preocupación por el "alto coste” que suponía disponer de software educativo en las escuelas, ahora la situación ha cambiado totalmente. Al tradicional y solitario ordenador de la secretaría del centro, se han añadido muchos otros más productos de donaciones, adquisiciones o renovaciones de dotación que van multiplicando el número de los equipos existentes. Además hay que considerar las posibilidades de los ordenadores portátiles que pueden repartirse cuando sean necesarios, y de la webcam que como otro medio o recurso más en el aula, puede facilitan la gestión administrativa, mejorar la comunicación interna del centro y abrir el centro a la sociedad.

Otro recurso que se utiliza en la gestión y que se ha desarrollado mucho en los últimos años es el software específico, que atiende tanto a la gestión económica como la organizativo-administrativa. Buena prueba de ello es que "el alto porcentaje de docentes que utilizan las TIC fuera del contexto del aula (para la planificación y la gestión administrativa) contrasta con el limitado porcentaje de profesorado que las usa dentro del contexto de la clase con los alumnos" (Area, 2008, 8). En realidad nos encontramos ante una serie de sistemas que integran, en su mayoría, una apuesta tecnológica de origen público o privado y que relaciona en mayor o menor medida una aplicación informática, un tipo de hardware y un website. Vamos a describir con más detalle algunos de estos sistemas:

a) En el caso del software proporcionado por las Administraciones educativas, si nos fijamos en el contexto autonómico valenciano, nos encontramos con el programa denominado GESCEN. Este programa, diseñado y actualizado por la Conselleria d'Educació de la Generalitat Valenciana, se puede encontrar en Gestión Informática de los Centros $^{[3]}$, (figura 1), donde se ofrece información de las distintas aplicaciones dedicadas a

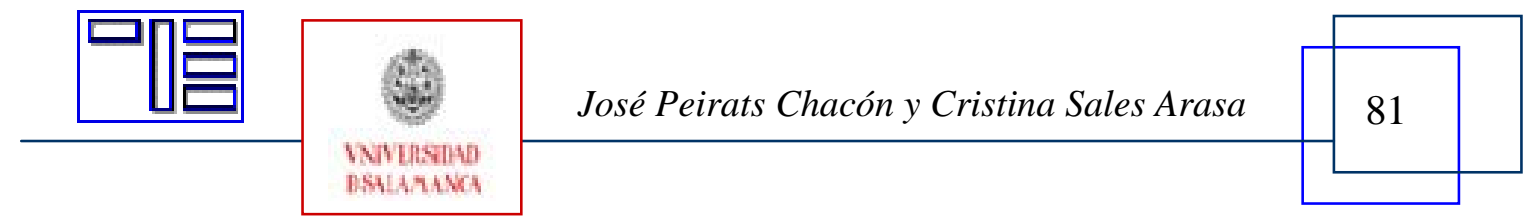


Revista Electrónica Teoría de la Educación.

Educación y Cultura en la Sociedad de la Información.

http://www.usal.es/teoriaeducacion

Vol. 10. No1. Marzo 2009

la gestión de los centros docentes, así como descarga de recursos, actualización de programas, foro donde preguntar y encontrar respuesta, preguntas frecuentes, etc.

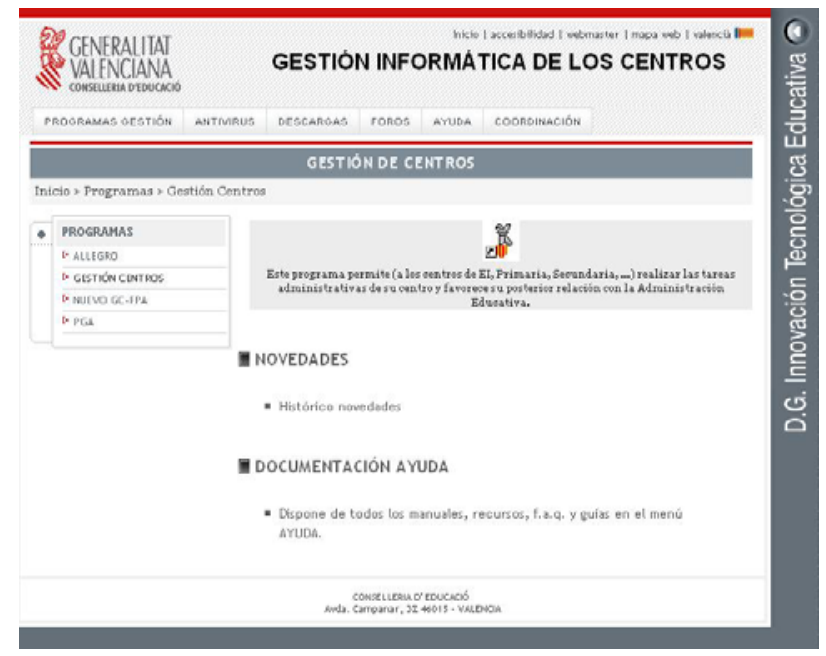

Figura 1: Web de Gestión Informática de Centros.

Es un sitio muy dinámico, comparado con otras webs de los distintos programas y servicios informáticos educativos, en continua actualización de la información y de las versiones de los programas de gestión con los que la Conselleria dota a los centros. Bajo nuestro punto de vista, estas páginas web trasladan la sensación de disponer de amplios apoyos y recursos tanto humanos como materiales, algo que contrasta llamativamente con la cantidad de críticas que ha recibido el GESCEN desde las secretarías de los centros a lo largo de su década de existencia. Este programa de gestión ha supuesto durante mucho tiempo una "intensificación del tiempo de trabajo" del docente, concretamente del secretario del centro -y que no hay que olvidar que en los centros de primaria es un maestro sin formación específica en conocimientos administrativos, de contabilidad o informáticos-, que se traduce en muchas horas dedicadas a su aprendizaje, debido a las grandes dificultades de manejo y a la cantidad de errores que todavía hoy genera.

Quizás por ello, recientemente se ha creado una red territorial de coordinadores. Son usuarios con experiencia en la utilización de los distintos aspectos del programa GESCEN, y cuya misión no es la de convertirse en un servicio técnico de apoyo sino que, en concreto pretenden:

- Acercar a los centros de la zona de influencia la ayuda, últimas novedades, información, formación, recogida de inquietudes, convocatoria de reuniones comarcales, etc.

- Disponibilidad de recursos y/o información sobre ellos: por correo electrónico a quienes lo soliciten, excepcionalmente por teléfono o presencial.

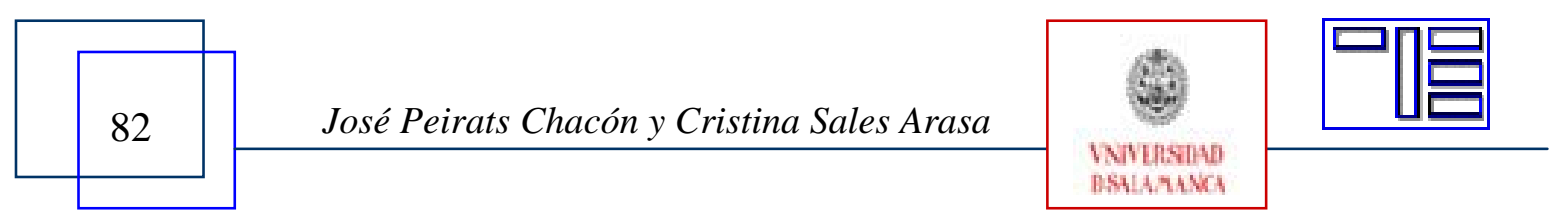




\begin{tabular}{|c|c|c|}
\hline 밈ㅁㅁ & $\begin{array}{l}\text { Revista Electrónic } \\
\text { Educación y Cultura en }\end{array}$ & $\begin{array}{l}\text { ducación. } \\
\text { la Información. }\end{array}$ \\
\hline & http://www.usal.es/teoriaeducacion & Vol. 10. $N^{\circ}$ 1. Marzo $200 \mathrm{~s}$ \\
\hline
\end{tabular}

- Servir de puente entre los centros y los coordinadores del proyecto, recoger propuestas e inquietudes sobre formación en un tema concreto del GESCEN y, sobre todo, de formación para realizar la Programación General Anual (PGA).

- Realizar reuniones formativas con los centros de la zona de influencia para enseñar aspectos nuevos o confusos sobre el GESCEN o sobre el programa de PGA.

Aunque consideramos que es una buena propuesta formativa de trabajo nos tememos que pronto se pueda desvirtuar, y por los motivos de siempre: la insuficiencia de los recursos oficiales, lo cual provoca que el éxito de la medida dependa de la voluntariedad de los docentes/coordinadores implicados. Y más cuando conocemos datos como el que se desprende de una investigación reciente de Mominó y otros $(2008,69)$ realizada en Catalunya: "entre las principales preocupaciones expresada por los responsables de las escuelas e institutos, no aparece el impulso y la difusión de las TIC”. Añadir más funciones, sin contraprestación horaria, a profesionales que ya desempeñan un trabajo no creemos que sea una medida muy apropiada para resolver las múltiples dudas y problemas que surgen alrededor de un programa de gestión que afecta a la organización de los centros docentes de la Comunidad Valenciana.

Recientemente han aparecido en la prensa local noticias destacadas que dan cuenta de la desaparición del GESCEN y su sustitución por el ITACA -Innovación Tecnológica para la Administración de Centros y Alumnos-. Un sistema formado por un superordenador con capacidad para 40.000 GB y formado por 25 servidores en red trabajando simultáneamente, una aplicación informática, una infraestructura informática con previsión del envío de un millón de SMS a padres y el almacenaje del expediente de un millón de alumnos, una página web para la consulta de notas, faltas y todo tipo de comunicaciones por los padres mediante contraseña. El sistema, a diferencia del anterior, desarrollado en su integridad por técnicos de la propia Conselleria, ha sido ofertado en concurso a la empresa privada.

En palabras de responsables de la Conselleria d'Educació ${ }^{[4]}$ "se sustituye un sistema basado en la descentralización de los datos, cada centro gestionaba los suyos en sus ordenadores y con el programa de gestión oficial, por otro en el que toda la información se maneja en red y se deposita en los servidores del ITACA". El trasvase de los datos que poseen todas las secretarías de los centros de la Comunidad al servidor, principal preocupación de los equipos directivos, las mismas fuentes han asegurado que lo realizarán técnicos del programa. Se pretende, por tanto, que para el próximo curso 2008-2009 se implante el nuevo sistema en todos los centros de infantil y primaria; realizando el proceso de matriculación, que se desarrolla en el mes de mayo del curso anterior, con el nuevo sistema. Si la previsión de recursos no ha sido la adecuada, y no encontramos en la literatura pruebas que demuestren la generosidad de las administraciones, este proce-

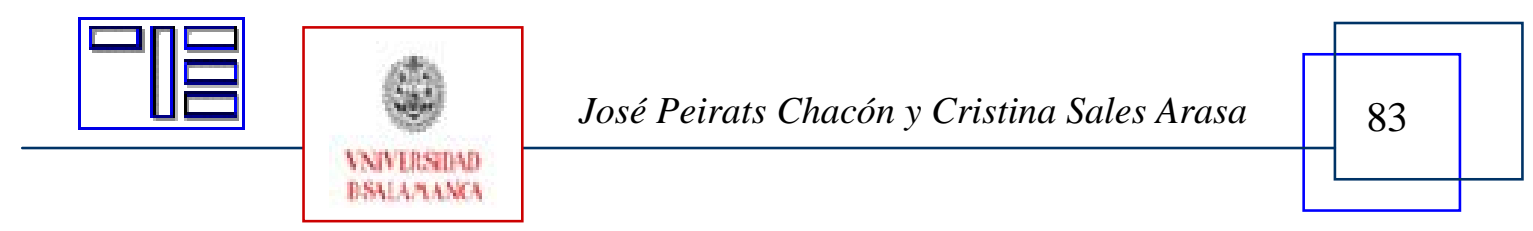


so tan importante y delicado que acontece en todos los cursos escolares desembocará, seguramente, en un bloqueo espectacular ${ }^{[5]}$.

A favor del nuevo sistema de gestión, se arguye la agilización de las labores administrativas, ya que el alumno llega a los centros con un número de expediente depositado en ITACA, se simplifica la elaboración del PGA al disponer la administración de los datos actuales de los centros, desaparece el envío y recepción de datos en soportes físicos y la consulta de datos a los centros por parte de Inspección y los distintos servicios de la Administración educativa. Tantos avances prometidos, en nuestra opinión, no consiguen eliminar la enorme preocupación ante un cambio tan radical en el ámbito administrativo y de gestión de los centros.

Sigue sin tenerse en cuenta en este proceso el envío de personal administrativo a los centros que no disponen de ellos, por tanto las labores de organización y control de los procesos administrativo-educativo como son la tramitación de becas, la puesta en marcha del comedor escolar, las matrículas de los alumnos, las bajas de los profesores, etc. y la introducción de los datos en el ITACA continuará recayendo sobre los hombros de unos responsables que hasta ahora ha gozado de muy pocas compensaciones. Sólo en este curso escolar, iqué coincidencia más casual!, han conseguido negociar los sindicatos con la Administración valenciana unas nuevas y sustanciosas retribuciones para los equipos directivos ¿tendrán algo que ver con el empeño de la Administración de implantar rápidamente el nuevo sistema?

Aunque nos hemos extendido considerablemente en esta comunidad, como botón de muestra de las políticas educativas autonómicas, también y dentro del ámbito público encontramos que la mayoría de las Administraciones territoriales desarrollan y suministran aplicaciones informáticas que cubren estas funciones. Ejemplos podemos encontrar en el programa Séneca para la gestión de centros docentes dependientes de la Junta de Andalucía $^{[6]}$, el Delphos (Sistema de Gestión para la Red de Centros Educativos Públicos) de la Junta de Comunidades Castilla-La Mancha, El Sistema d'Administració i Gestió Acadèmica (SAGA) en Cataluña ${ }^{[7]}$, e incluso el Ministerio de Educación ${ }^{[8]}$ también facilita las aplicaciones de gestión económica GECE 2000, el Programa Escuela para infantil y primaria y el IES 2000 para secundaria.

b) En el caso de sistemas combinados de software, hardware y web entramos de lleno en el terreno comercial, donde encontramos variadas empresas que ofrecen sus productos con precios que publicitan asequibles a cualquier centro, al ofrecerlos por módulos independientes; en función del mayor o menor número que se contrate resultará el costo final. Veamos una muestra de los servicios que ofrecen algunas empresas:

- Mentor Vox Apli ${ }^{[9]}$ es un sistema formado por una aplicación y una web que enfatiza la comunicación con las familias. Para ello genera un canal para compartir la información que se genera día a día: notas, absentismo escolar, incidencias, horarios, comedor, circulares, etc. y permite el acceso por Internet y por teléfo-

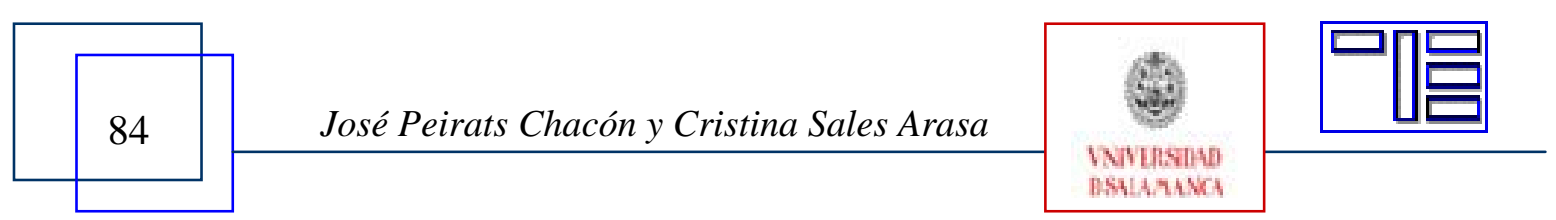




\begin{tabular}{|c|c|}
\hline ص & $\begin{array}{l}\text { Revista Electrónic } \\
\text { Educación y Cultura en }\end{array}$ \\
\hline & http://www.usal.es/teoriaeducacion \\
\hline
\end{tabular}

no. La entrada de datos se realiza directamente en la aplicación o mediante un escáner que lee la información en soporte papel del profesorado, aunque próximamente incluirán una PDA.

- En Excellence Software desarrollan una aplicación informática denominada Eduexcellence $^{[10]}$ que no necesita de otros artefactos tecnológicos para el profesor. Simplemente éste cumplimenta una hoja con la lista de clase y un código de barras de membrete con los comentarios que considera oportuno y con los datos administrativos básicos: faltas, expulsiones, retrasos, etc. Una vez entregado el documento en administración se escanea junto a los del resto de sus compañeros. Dispone de varios módulos, entre ellos los de comunicados, comedor, tutorías y pretende ofrecer la información al día y permanentemente para toda la comunidad educativa; en el caso de los padres a través del envío de SMS puntuales y de fichas semanales del rendimiento de los hijos. Se diferencian del resto de sistemas de gestión en que al profesor no se le exige un trabajo suplementario de emisión de informes o manejo de tecnologías complementarias como el PDA.

- También relacionado con la gestión encontramos en el mercado otro producto: el Sistema de Gestión Docente (SGD) ${ }^{[11]}$, conocido popularmente en la Comunidad Valenciana con el mismo nombre que el juguete infantil "Tamagochi”, y que parece imponerse en muchos centros a tenor del elevado número que se relacionan en la web destinada a las consultas externas por las familias. Este sistema está compuesto por unidades personales para cada profesor, unidad central donde se almacenan las unidades y se recogen los datos, y el programa que gestiona los datos que introducen los profesores. Permite realizar una serie de tareas relacionadas con el control del absentismo de los alumnos, la entrada de calificaciones, el seguimiento de alumnos y profesores, y la comunicación entre directivas y profesores. El proceso se realiza de forma ágil y cómoda en la unidad personal y elimina la engorrosa cumplimentación de estadillos e informes.
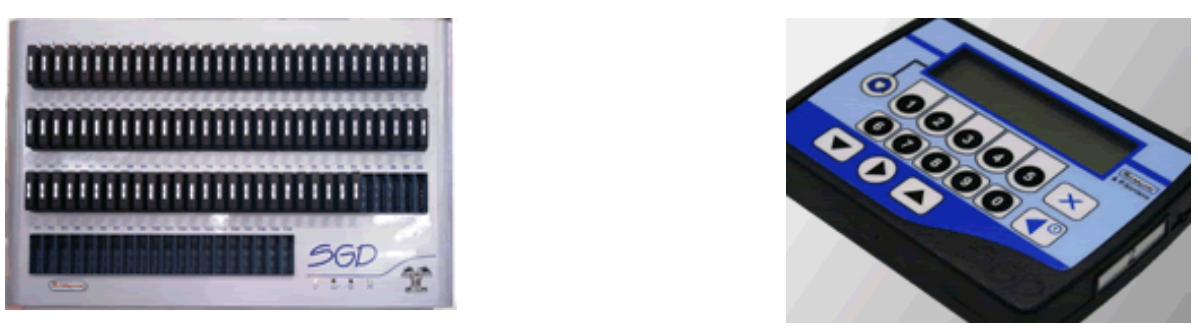

Figura 2: Unidad central y personal del SGD (Consulta el 14/04/08).

Este último sistema complementa pero no sustituye al programa oficial de gestión del centro suministrado por la Administración educativa y los datos pueden ser tanto importados como exportados al programa oficial, según reza en la publicidad.

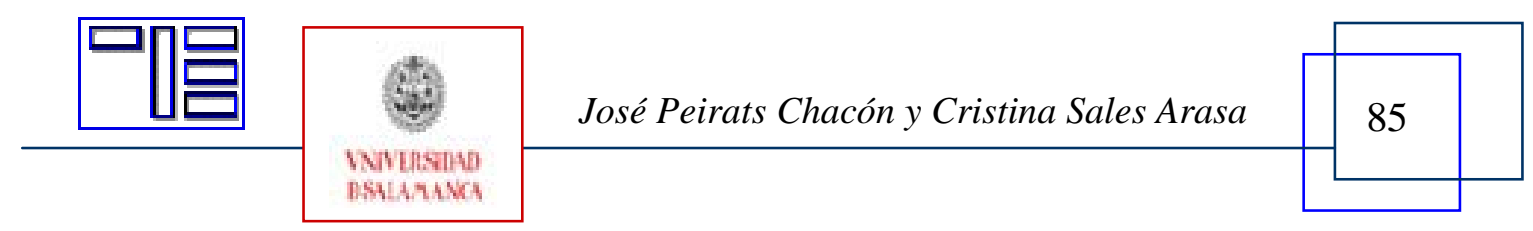


- También EDUCANET, con el programa IESFácil o SecreFácil ${ }^{[12]}$ programa informático multi-capa, es decir que utiliza un Servidor de Aplicaciones que comunica con los datos y con los puestos, la información se almacena sin necesidad de compartir carpetas. Existe la posibilidad de instalación en multitud de equipos dentro de la red del Centro o fuera de la misma (a través de Internet) en una única Base de Datos y sin afectar el rendimiento del Sistema. Permite el envío masivo de SMS pero no el acceso de las familias vía Internet.

Bajo nuestro punto de vista, en todos estos sistemas advertimos en su contra que, al ser productos comerciales, acarrean el inconveniente de su elevado precio, al que deben hacer frente los centros docentes con los tradicionalmente escasos presupuestos ordinarios.

c) De carácter shareware o freeware también encontramos varios productos en la red. Desde los ofrecidos en plataformas de apoyo al sector educativo, como es el caso de EDUCARED que ofrece un sistema de gestión (DocCF, Gestión Escolar ${ }^{[13]}$ ) que se puede descargar gratuitamente desde sus páginas. O el ofrecido por SoftAula ${ }^{[14]}$, empresa comercial que dispone de productos más completos y de pago pero también de una versión gratuita. Aunque el listado que podemos encontrar en la red es muy extenso: Artesoft Escuela, Horarios Escolares, Idesoft, MZT Sistemas, NetSupport School, etc.

Y por último, fijándonos en las migraciones que últimamente se están produciendo hacia el software libre podemos identificar alguna tentativa en este tema. Así se encuentra a nuestra disposición el Proyecto SIESTTA ${ }^{[15]}$, con dos grandes módulos: docentes y familias; y con dos formas de uso diferente: registrarse en su página web y utilizarlo a través de Internet mediante un portátil, o descargar el código fuente e instalarlo en un servidor web propio del centro educativo.

\section{3.- INTERACCIONES EMERGENTES ENTRE TECNOLOGÍAS DE GESTIÓN Y CENTROS DE SECUNDARIA.}

Después del avance tecnológico experimentado en los últimos años encontramos a los centros educativos sobrados de ordenadores, con sistemas informáticos en denodada lucha entre el código libre o en código propietario y de docentes apresurada e improvisadamente reciclados como informáticos; aunque, en la realidad, algunos centros podemos considerarlos como cementerios tecnológicos. En esa perspectiva hemos constatado, en algunas de las entrevistas con técnicos de informática de la Administración valenciana, un cierto tono de desencanto hacia el funcionamiento general del programa de informática de los centros y hacia su desinterés en la aceptación y aplicación de algunas de las medidas organizativas impulsadas desde la Administración. Afirman, por ejemplo, que en algunos centros de secundaria, existe una falta de voluntad de muchas juntas directivas para apoyar material y organizativamente los esfuerzos de muchos profesores empeñados en integrar los recursos informáticos; y, paradójicamente, también nos han desvelado la aparición de otros centros en los que, motivados por la escasez de matrícu-

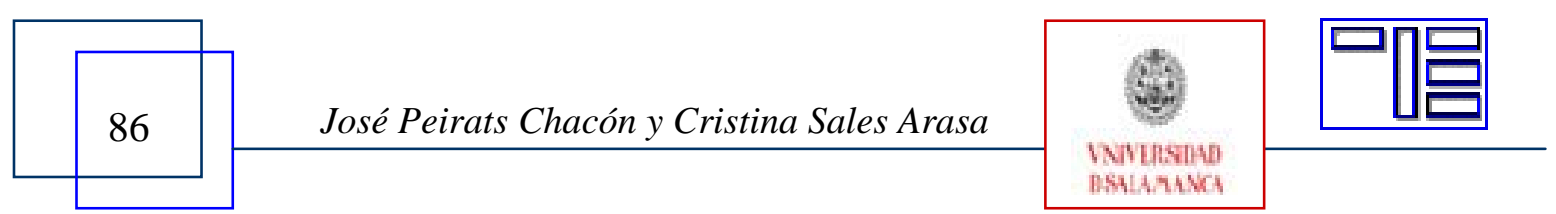


las de alumnos, se están volcando en la potenciación de los recursos informáticos en la formación como medio para atraer al alumnado. En este orden de cosas, el espectacular desarrollo del software está posibilitando la aparición de aplicaciones que buscan ayudar al docente en el trabajo burocrático, los conocidos programas de gestión; sin embargo debemos alertar ante una serie de consecuencias que por su utilización se están produciendo, veamos algunas de ellas.

En primer lugar encontramos los crecientes mecanismos de control relacionados con los artefactos asociados a los programas de gestión. Muchos docentes ante la facilidad con la que se agilizan con dichos programas algunas de las engorrosas tareas administrativas -notas, faltas, amonestaciones, etc.- conceden, a cambio, sin pensárselo dos veces el control instantáneo sobre su labor a los órganos directivos; como nos decía un profesor: "en este tema como en otros nos dejamos llevar, básicamente lo que aporta es comodidad porque ahorra trabajo". En este sentido, señalamos que la actividad de "fichar" al comenzar la jornada laboral, tan usual en las empresas del mundo laboral, no se ha propuesto en los centros de secundaria, lo que sin duda hubiera provocado grandes reticencias por parte del profesorado. Sino que mediante el reclamo de las ventajas que suponen la utilización de los artefactos de gestión, se ha sustituido indirectamente por la retirada de la unidad personalizada (el citado Tamagochi) en el panel de control; instalado, por lo general, confortablemente en las salas de descanso de los profesores. Desde ese preciso momento y sin que sea consciente el profesorado, como así nos lo han confirmado en las entrevistas realizadas, el equipo directivo es conocedor, a través de la aplicación que gestionan los artefactos tecnológicos, de quién y cuándo la ha retirado y cómo o para qué la ha utilizado. No es menester recordar el argumento del libro 1984 de Orwell, pero sí que nos hace pensar un poco sobre las motivaciones implícitas que puedan existir tras el acusado interés hacia la utilización de estos artefactos tecnológicos.

No se puede negar que agiliza tareas administrativas a los docentes pero al mismo tiempo también es un sistema de control de la jerarquía, en este caso Jefe de Estudios, tanto hacia el profesorado como hacia el alumnado; y del mismo modo, aunque en menor medida, del tutor de los grupos de alumnos como de los profesores en general. Sin embargo, en la práctica, algunas veces los tutores no ejercen sus funciones, así nos lo decían: "no hay que ser tan rigurosos", "no me he acordado de sacar el informe de la semana anterior", y no revisan en el programa de gestión las incidencias acaecidas, ni toman las decisiones pertinentes o no esclarecen con los alumnos los hechos reflejados: expulsiones, amonestaciones, retrasos, faltas, etc. "a veces pasan los días y la marcha normal o las nuevas incidencias te hacen olvidar lo pendiente”. O, preocupados por la puntualidad, entran en las aulas sin pasar recoger el artefacto de control.

Por otro lado encontramos que la convergencia de múltiples tecnologías se refleja en la penetración de más y más artefactos que exigen una alta formación permanente y especializada, pero que descuida o simplemente no contempla aspectos como el desarrollo de una conciencia crítica en los alumnos, o la estructuración de un sistema consistente de símbolos y valores que les permita descodificar acertadamente los mensajes que las

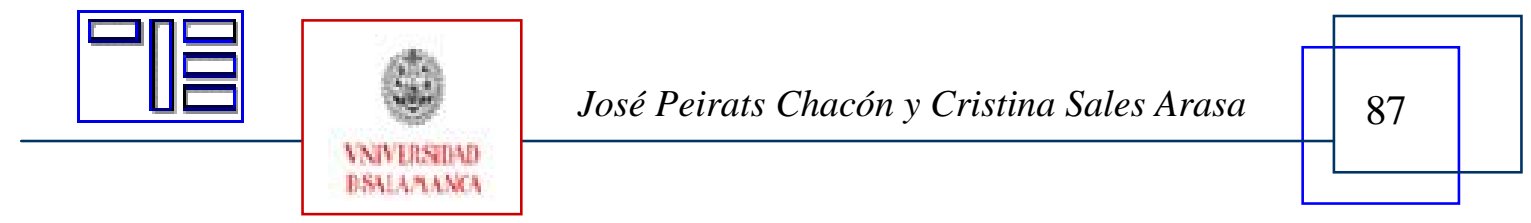


Revista Electrónica Teoría de la Educación.

Educación y Cultura en la Sociedad de la Información.

http://www.usal.es/teoriaeducacion

Vol. 10. No1. Marzo 2009

tecnologías transmiten. En definitiva nos sumamos a Torres (1994), cuando afirma que el recurso tecnológico es necesario en esta sociedad informatizada porque nos permite informarnos, formarnos, comunicarnos y explayarnos y porque la escuela todavía conserva funciones en la educación. Suyo es el deber de enseñar no sólo los componentes instrumentales sino también de alertar sobre los intereses que se esconden tras estos artefactos y de transmitir valores morales auténticos y duraderos; en definitiva, una educación verdaderamente comprometida con valores de democracia, solidaridad y crítica.

Y en este aspecto tienen mucho que aportar las familias de los alumnos ya que gran parte de la efectividad del trabajo escolar depende de cómo se rediseñen las relaciones con las familias, y más cuando un profesor confesaba que "una de las paranoias que tienen o tenemos muchos de los profesores es precisamente el trato con los padres". Por este motivo, pensar la participación de las familias, las formas de comunicación con ellas, la canalización de sus inquietudes y demandas constituye una gran responsabilidad para los centros docentes. En estos momentos muchas de las tecnologías implicadas en la gestión de lo centros se están utilizando en la comunicación hacia las familias; básicamente en la información a los padres sobre faltas, retrasos, expulsiones, expedientes disciplinarios, evaluaciones de sus hijos. Pero algunas de estas tecnologías se gestionan desde el ámbito de la empresa privada a través de las plataformas de consulta; los centros facilitan unos datos que posteriormente, al margen de las instituciones, se alojarán en webs que puedan acceder, eso sí mediante contraseña, los padres de los alumnos. Otro peligro que últimamente se está advirtiendo se encuentra en la proliferación de software de intercambio de archivos en ordenadores de empresas e instituciones, el acceso a información sensible puede ser facilitado por estos medios a manos externas. Y, ante estas prácticas nos preguntamos hasta qué punto es permisible que datos confidenciales se trasvasen a otros ámbitos, ¿quién es responsable de la custodia de los datos de los alumnos en los centros?, ¿no es una de las funciones que desempeñan los secretarios?, ¿se estará incurriendo en alguna ilegalidad más o menos encubierta por la firma de las autorizaciones de los padres?

Es evidente en este aspecto, fuertemente relacionado con los intereses privados, que existe un desconocimiento generalizado de la legislación sobre protección de datos, aunque en este tema no sólo está afectado el sector educativo. A lo largo de 2006 la Agencia Española de Protección de Datos inspeccionó varias decenas de centros docentes en los que, desde siempre, se han manejado información delicada o confidencial de las familias. Las conclusiones han sido desalentadoras: datos "excesivos", sin seguridad suficiente para protegerlos, cesión de datos sin consentimiento expreso, desconocimiento hacia la custodia y destrucción, etc. calificando con un suspenso la protección de los datos recabados. En 2007 han sido abiertos por la agencia 21 expedientes por filtraciones de datos personales a Internet. Urge, por tanto, la necesidad de esclarecer los papeles de cada uno de los sectores implicados y este proceso "debería ayudar a mantener la confianza de los padres en las decisiones que se toman, incluida la organización y administración de la cotidianeidad escolar y las políticas de exigencia con sus incentivos y sanciones” (Carriego, 2006). Otros estudiosos como Valero Torrijos (2007) han estu-

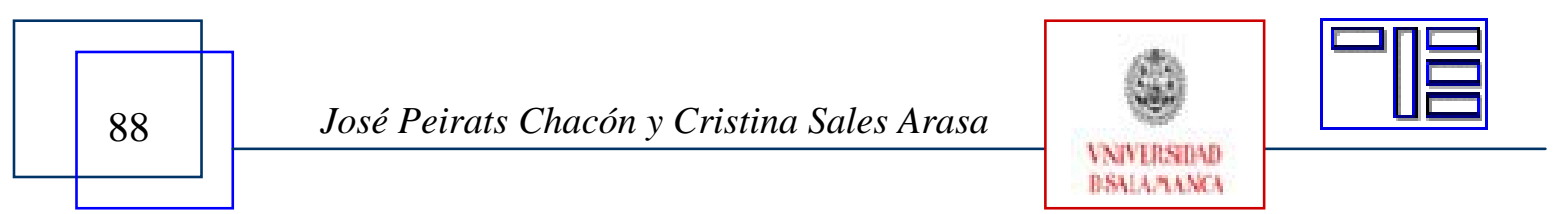


diado el campo desde la óptica de sus implicaciones jurídicas para las Administraciones Públicas y han señalado la trascendencia que puede llegar a tener el Derecho a la hora de obstaculizar y/o impulsar los proyectos de modernización tecnológica, y han advertido sobre la problemática específica que, desde el punto de vista de la responsabilidad patrimonial, supone el uso de medios informáticos y telemáticos en la actividad administrativa.

Y también en este tema tienen algo que decir los jóvenes que, ante la presencia de artefactos como el "Tamagochi”, cuya utilización perciben como instrumento de control de sus acciones, y la promulgación generalizada de medidas disciplinarias en los centros contra la utilización de los teléfonos móviles, se deben sentir atacados por un sistema tecnológico que no es el suyo y por un sistema de valores que comparten padres y profesores pero que no coinciden con los propios.

Para finalizar este apartado debemos resaltar que existe, además, un cierto problema radicado en el coste de los sistemas de gestión. Si tenemos un sistema doble en el que coexisten un sistema de gestión dotado por la Administración y otro que complementa, ayuda o suple aspectos que no cubren el anterior, las dificultades se pueden multiplicar. Por un lado encontramos que la carestía del sistema complementario y comercial aumenta o disminuye en función de la cantidad de módulos que se contrate, es decir se puede ajustar a las disponibilidades de los centros, pero siempre serán unos recursos económicos que se detraerán de otras necesidades existentes. Por otro observamos, como contrapartida, que en determinados lugares, al reconocerles valor a las funciones que cubren, estos dispendios son asumidos por municipios ${ }^{[16]}$, asociaciones de padres o, incluso, subvencionados por otras empresas. En realidad, nos estamos encontrando ante otro intento de entrada del capital privado en el "negocio" de la educación y que no es la primera ni, por desgracia, la última que soportará la escuela pública.

\section{CONCLUSIONES.}

Software de gestión: ¿aplicaciones para informar a las familias y ayudar a los docentes o para controlar a profesores y alumnos? Entre estas contradicciones se mueve la convergencia en los centros de estas tecnologías que mueven ciertas complicidades en los estamentos de poder pero también muchas inquietudes entre el profesorado. En este caso nos encontramos que el recurso tecnológico está al servicio del docente facilitándole el trabajo burocrático que, en numerosas ocasiones, entorpece su labor aunque a costa de perder cotas de libertad y autonomía profesional, al ser un artefacto que proporciona a la dirección del centro el control al instante de sus quehaceres.

En este campo fértil y abierto a la competencia encontramos una proliferación de empresas del ámbito privado o comercial dedicadas al desarrollo de aplicaciones y de herramientas al servicio de la gestión de los centros. Lo que indica negocio y futuro para sus inversiones que ya se evidencia en la contrapublicidad que algunas realizan intentando desvirtuar las ventajas que otras entidades alardean: si unas brindan los escáneres

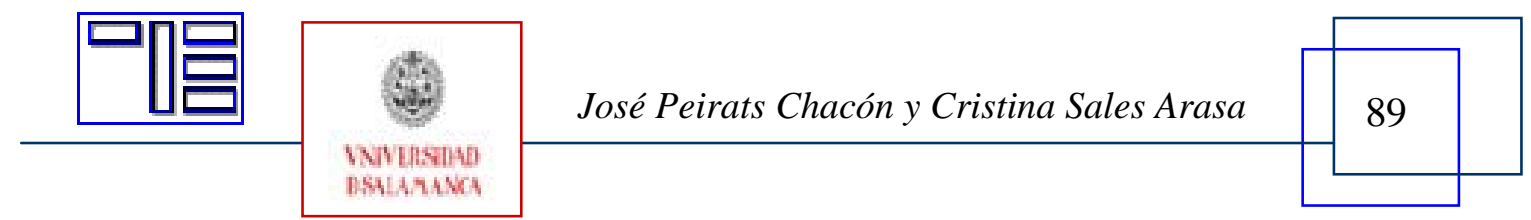


y las consejerías de los centros como instrumentos ideales para la entrada de la información, otras opinan lo contrario y proponen los PDA o los "sitios" en red como mejor sistema. En esta competencia tecnológica, ¿̇e sumarán de la misma manera las administraciones educativas?

Se pretende la información permanente y, por tanto, el control continuo y al instante de profesores y alumnos por parte de padres, equipos directivos y administración educativa. Al mismo tiempo, al trasladar datos de los centros a plataformas alojadas en servidores independientes y mantenidos por personal de las empresas suministradoras del sistema de gestión se puede estar incurriendo en una ilegalidad, si se ponen sin autorización expresa datos de las familias de los alumnos de carácter confidencial en manos de empresas privadas al margen de la institución educativa. En este aspecto cabe señalar también y como problema colateral la desigual adaptación de la administración a la sociedad digital, en la que brillan con gran intensidad determinados organismos como la Agencia Tributaria y palidecen casi todos los demás.

Para finalizar y, como en otras ocasiones, seguiremos defendiendo las variaciones en la organización para innovar pero no para controlar (Peirats, 2008). Las tecnologías que entran en la escuela son producto de su tiempo y se han de acomodar junto al resto de recursos que se poseen y, eso sí, en función de las directrices que emanan de los proyectos de centro. Sin perder de vista, como así nos lo recordaba un profesor entrevistado, que los verdaderos problemas a los que se enfrenta la escuela son humanos, no los resuelve el Tamagochi sino que requieren una gestión humana. Los nuevos recursos de gestión, en la medida que ayudan o agilizan las penosas actividades burocráticas, también tienen su sitio en la escuela de la Sociedad del Conocimiento que pretendemos construir; sin embargo, debemos seguir denunciando a los instrumentos y a los sujetos que sólo los utilizan en un sentido restrictivo, para la férrea vigilancia y la emisión de medidas disciplinarias. O quizás hayamos de plantearnos que este panorama sea uno de los efectos visibles de las TIC que Kerr vaticinara en 1996, efectos que según él, se iban a dar sobre todo en la forma de organizarse los centros, en la distribución de poder entre sus miembros y en las relaciones del centro con la comunidad. En cualquier caso educar en y para la libertad y la democracia exige compromisos personales más elevados que están muy por encima de falsas ventajas o espejismos del reclamo tecnológico.

\section{5.- REFERENCIAS.}

AREA, M. (2008): Una breve historia de las políticas de incorporación de las tecnologías digitales al sistema escolar en España. En Quaderns Digitals, 51, 1-12. http://www.quadernsdigitals.net/index.php?accionMenu=hemeroteca.VisualizaA rticuloIU.visualiza\&articulo_id=10454 (Consultado el 14/03/08).

CARRIEGO, C. (2006): Gestionar una escuela comprometida con las demandas de su tiempo. En Revista Iberoamericana de Educación, 39/2 http://www.rieoei.org/1421.htm (Consultado el 12/02/08).

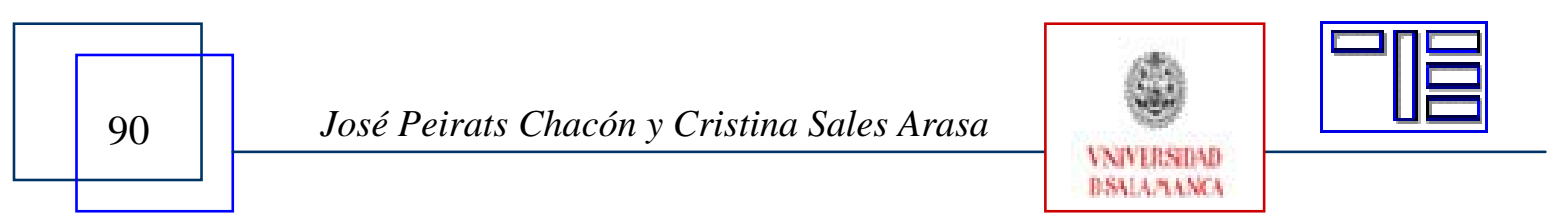


DESSLER, G. (2001): Management: Leading People and Organizations in the 21st Century. New Jersey, Prentice Hall.

EZPELETA, J. (1997): Algunos desafíos para la gestión de las escuelas multigrado. En Revista Iberoamericana de Educación, 15. En http://www.rieoei.org/oeivirt/ rie15a04.htm (Consultado el 08/04/08).

JARES, X. R. (1997): El lugar del conflicto en la organización escolar. En Revista Iberoamericana de Educación, 15. En http://www.rieoei.org/oeivirt/rie15a02.htm (Consultado el 08/04/08).

KERR, S. T. (1996): Toward a Sociology of Educational Technlogy. En JONASSEN, D. (Ed.): Handbook of Research for Educational Communications and Technology. New York, McMillan, 143-170.

LLORENS I CERDÀ, F. (2003): Tecnologías del conocimiento en Educación. En Quaderns Digitals. http://www.quadernsdigitals.net/index.php?accionMenu=boletines.Visualiza BoletinIU.visualiza\&boletin_id=15 (Consultado el 08/04/08).

McFARLANE, A. (2001): El aprendizaje y las tecnologías de la información: experiencias, promesas, posibilidades. Madrid, Santillana.

MOMINÓ, J. M.; SIGALÉS, C. y MENESES, J. (2008): La escuela en la sociedad Red. Internet en la Educación Primaria y Secundaria. Barcelona, Ariel.

PEIRATS, J. (2008): Variaciones organizativas generadas por las Tecnologías de la Información. Un estudio en los centros de Primaria de la Comunidad Valenciana. Valencia, Universitat de València.

RAMÍREZ, L. H. (2004): Los nuevos desafíos de la gerencia educativa. En Educación y Educadores, 7, 113-136. Disponible en:

http://biblioteca.unisabana.edu.co/revistas/index.php/eye/article/view/319/422 (Consultado el 02/04/08).

TORRES, J. (1994): Globalización e interdisciplinariedad: el curriculum integrado. Madrid, Morata.

VALERO TORRIJOS, J. (2007): El régimen jurídico de la e-Administración. El uso de medios informáticos y telemáticos en el procedimiento administrativo común. Granada, Editorial Comares.

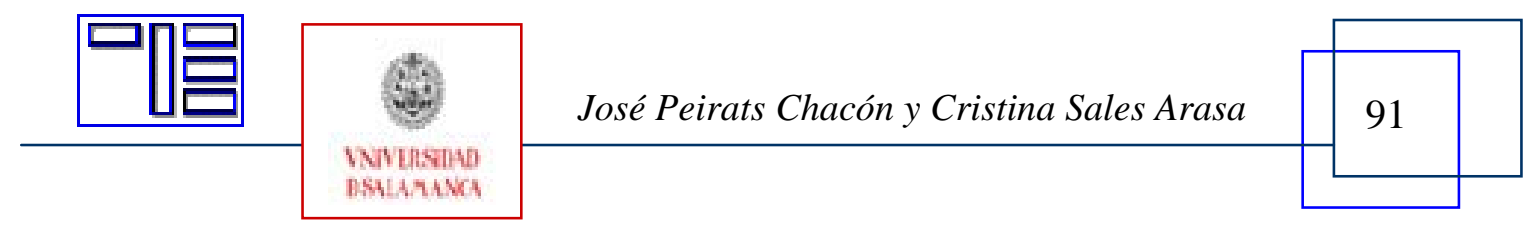


Revista Electrónica Teoría de la Educación.

Educación y Cultura en la Sociedad de la Información.

http://www.usal.es/teoriaeducacion

Vol. 10. No1. Marzo 2009

\section{Notas:}

${ }^{[1]}$ El 31 de diciembre vence el plazo para cumplir la ley de acceso electrónico de los ciudadanos a las administraciones.

[2] “Currículum, Recursos e Instituciones Educativas”, adscrito al Departamento de Didàctica i Organització Escolar de la Universitat de València.

${ }^{[3]}$ En http://www.edu.gva.es/soportegc/v_cas/gc.htm (Consulta el 12/04/08).

${ }^{[4]}$ Pronunciadas por Sofia Bellés, Directora General de Innovación Tecnológica Educativa de la Generalitat Valenciana (Levante EMV, 11/03/08).

[5] Estas palabras las escribimos hacia finales del primer trimestre de 2008; el 20 de junio la Directora General de Innovación Tecnológica Educativa paralizó el proceso de implantación del ITACA (carta publicada en http://www.edu.gva.es/webitaca/es/index.htm, consultada el 24/06/08), estableciendo un período de pilotaje con 75 centros en el primer trimestre del próximo curso y la incorporación graduada del resto.

[6] En https://www.juntadeandalucia.es/educacion/seneca/seneca/jsp/pag_inicio800.html (Consulta 12/04/08).

${ }^{[7]}$ En http://www.xtec.net/gestcent/saga-1/guia.htm> (Consulta 12/04/08).

${ }^{[8]}$ En http://gestioncentros.cnice.mec.es (Consulta 12/04/08).

${ }^{[9]}$ En http://www.mentorvox.com (Consulta 14/04/08).

${ }^{[10]}$ En http://www.eduexcellence.es/index.htm (Consulta 13/04/08).

${ }^{[11]}$ En http:/www. tecnausa.com y http://www.sgdweb.com (Consulta 12/04/08).

${ }^{[12]}$ En http://www.iesfacil.com (Consulta 14/04/08).

${ }^{[13]}$ En http://www.educared.net/educared/visualizacion/jsp/_software_educativo/Extra

curri_culardetalle.jsp?idapr =12_1197_esp_1_\&idArticulo=1978 (Consulta 13/04/08).

${ }^{[14]}$ En http://www.softaula.com (Consulta 14/04/08).

${ }^{[15]}$ En http://siestta.org (Consulta 14/04/08).

${ }^{[16]}$ En el IES "Albal” de Albal (Valencia) los gastos son asumidos por los Ayuntamientos de Albal y Beniparrel, el AMPA asume los gastos de los SMS (Las Provincias, 3 de febrero de 2006).

Para citar el presente artículo puede utilizar la siguiente referencia:

PEIRATS CHACÓN, José y SALES ARASA, Cristina (2009). Inquietudes y complicidades en el uso del software de gestión de centros educativos. En SAN MARTÍN ALONSO, A. (Coord.) Convergencia Tecnológica: la producción de pedagogía high tech [monográfico en línea]. Revista Electrónica Teoría de la Educación: Educación y Cultura en la Sociedad de la Información. Vol. 10, $\mathrm{n}^{\circ}$ 1. Universidad de Salamanca [Fecha de consulta: $\mathrm{dd} / \mathrm{mm} / \mathrm{aaa}]$.

http://www.usal.es/ teoriaeducacion/rev_numero_10_01/n10_01_peirats_sales.pdf ISSN: 1138-9737

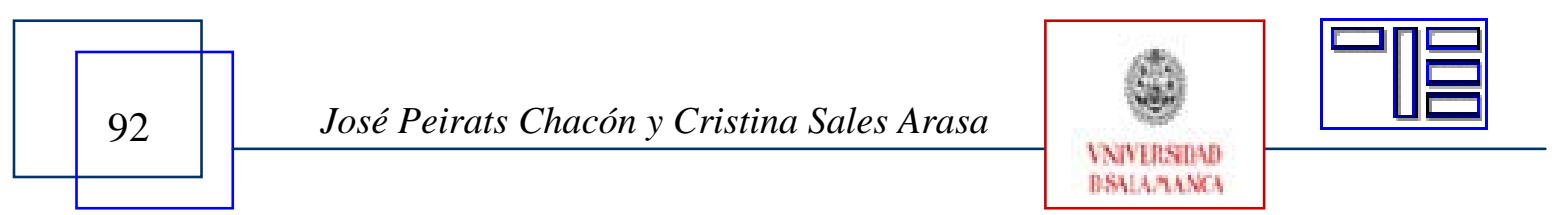

